\title{
The Linewidth Enhancement Factor in a Microcavity Brillouin Laser
}

\author{
Zhiquan Yuan, ${ }^{\dagger}$ Heming Wang, ${ }^{\dagger}$ Lue We, ${ }^{\dagger}$ Maodong Gao, and Kerry Vahala* \\ T. J. Watson Laboratory of Applied Physics, California Institute of Technology, Pasadena, California 91125, USA \\ *vahala@caltech.edu
}

\begin{abstract}
Amplitude-phase coupling can enhance the linewidth of regenerative oscillators and lasers. Here, in Brillouin lasers, the coupling is shown to originate from phase mismatch. Linewidth enhancements as large as 50× are measured. (c) 2021 The Author(s)
\end{abstract}

Microcavity-based stimulated Brillouin lasers (SBLs) have received considerable attention in recent years. Their compact size, high efficiency, flexible operating wavelength and ability to be chip integrated makes them of great interest for radio-frequency synthesizers, ring-laser gyroscopes and high-coherence sources. The fundamental linewidth of SBLs is dramatically increased relative to the conventional Schawlow-Townes (ST) linewidth on account of phonon participation in the lasing process [1]. In this work, we show that SBL linewidth is also increased by the linewidth enhancement factor $\alpha$ which characterizes amplitude-phase coupling of the field [2]. While this effect is well known in semiconductor laser systems [3], SBLs derive gain through a parametric process where scattering of an optical pump into a Stokes wave from an acoustic phonon must be phase-matched [4]. Therefore, in contrast to semiconductor lasers, it is shown that phase mismatch causes a non-zero $\alpha$ factor in SBLs. Measurements of SBL frequency noise are used to determine $\alpha$ versus controlled amounts of phase mismatch, and the results are in good agreement with theory.

Amplitude-phase coupling occurs when the real and imaginary parts of the optical susceptibility (equivalently refractive index and gain) experience correlated variations subject to a third parameter. The $\alpha$ parameter is defined as the ratio of the real to imaginary parts of such variation [3]. With a non-zero $\alpha$-parameter, noise that normally couples only into the laser field amplitude can also couple into the phase and therefore causes linewidth enhancement. The $\alpha$-factor enhanced fundamental SBL linewidth $\Delta v_{\mathrm{SBL}}$ is $\Delta v_{\mathrm{SBL}}=\Delta v_{0}\left(1+\alpha^{2}\right)$, where $\Delta v_{0}$ is the conventional $(\alpha=0)$ linewidth [1]. In the SBL system, optical pumping at frequency $\omega_{\mathrm{P}}$ on a cavity mode causes a Lorentzian-shaped Brillouin gain spectrum, which is frequency down-shifted by the phonon frequency $\Omega$ (Brillouin shift frequency) relative to $\omega_{\mathrm{P}}$ (Fig. 1a). Laser action at frequency $\omega_{\mathrm{L}}$ is possible when a second cavity mode lies within the gain spectrum, which requires that $\Delta \omega \equiv \omega_{\mathrm{P}}-\omega_{\mathrm{L}}$ is close in value to $\Omega$. Perfect phase matching corresponds to laser oscillation at the peak of the gain (i.e., $\Delta \omega=\Omega$ ). Also shown in Fig. 1a is the refractive index spectrum associated with the gain spectrum according to the Kramers-Kronig relations. It is apparent that $\alpha$ (the ratio of variation of real to imaginary susceptibility) will be zero at the phase matching condition, while it increases with increased frequency detuning relative to the gain center.

The linewidth enhancement factor can be expressed using two equivalent mismatch quantities relative to perfect phase matching, $\alpha=2 \delta \Omega / \Gamma=2 \delta \omega / \gamma$. In the first equality, phonon mode detuning $\delta \Omega \equiv \Omega-\Delta \omega$ is normalized

(a)

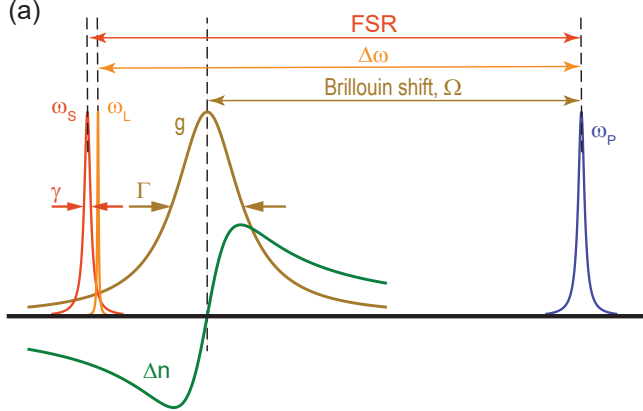

(b)

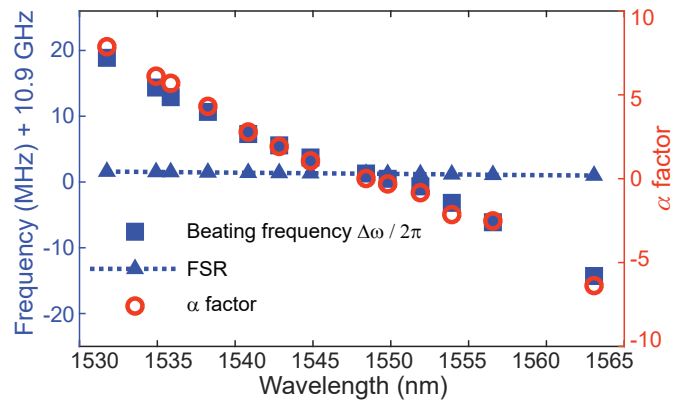

Fig. 1. (a) Brillouin gain process in the frequency domain. Blue (red) curve refers to pump (Stokes) cavity mode at frequency $\omega_{\mathrm{P}}\left(\omega_{\mathrm{S}}\right)$. Orange curve refers to the SBL laser signal at frequency $\omega_{\mathrm{L}}$. Brown and green curves correspond to gain $(\mathrm{g})$ spectrum and refractive index $(\Delta \mathrm{n})$, respectively. (b) The extrapolated beating frequency (squares) and FSR (triangles) are plotted versus wavelength. The calculated $\alpha$ factor (red circles) is plotted versus wavelength. The Brillouin gain center occurs at around $1548 \mathrm{~nm}$ where $\mathrm{FSR}=\Delta \omega$. 

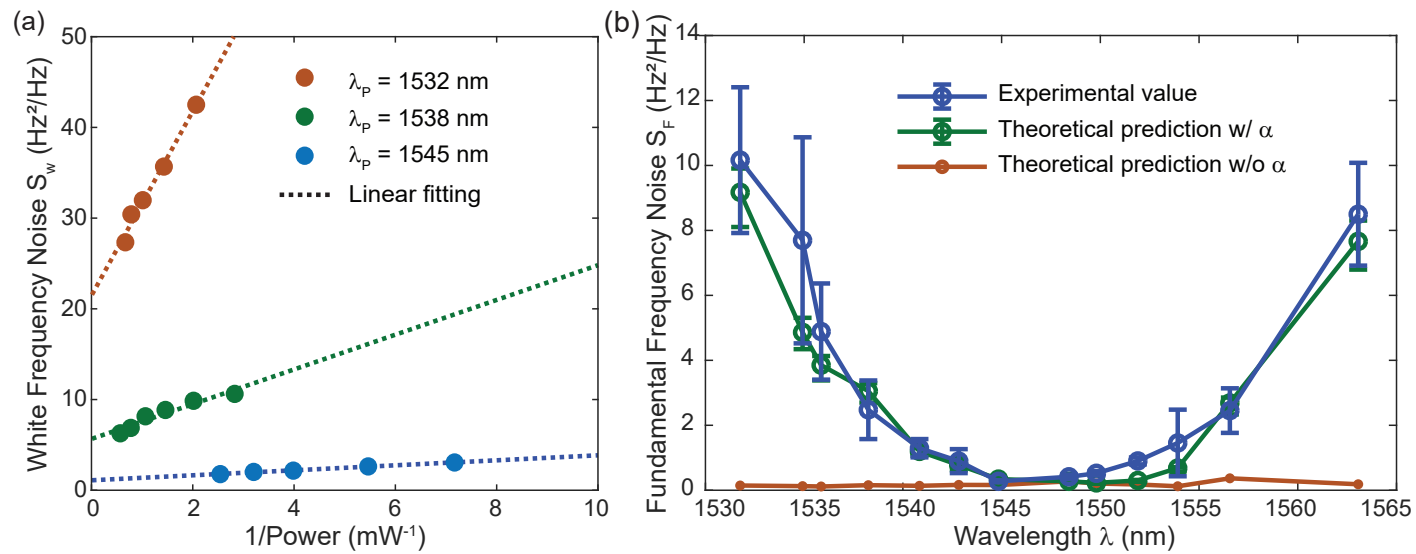

Fig. 2. (a) SBL white frequency noise $S_{\mathrm{w}}$ is plotted versus the reciprocal of SBL output power. A linear fitting is applied to determine $S_{\mathrm{F}}$ from the slope, and then plotted in the panel (b). (b) Measured SBL fundamental frequency noise $S_{\mathrm{F}}$ (blue); theoretical $S_{\mathrm{F}}\left(S_{\mathrm{F}}=\Delta v_{\mathrm{SBL}} /(2 \pi)\right)$ prediction (green) with $\alpha$ obtained from Fig. $1 \mathrm{~b}$; and non-enhanced $S_{0}\left(S_{0}=\Delta v_{0} /(2 \pi)\right)$ prediction (red); all plotted versus pump wavelength and normalized to $1 \mathrm{~mW}$ output power.

by $\Gamma$, the Brillouin gain bandwidth (the phonon decay rate). In the second equality, optical mode detuning $\delta \omega \equiv$ $\Delta \omega-$ FSR (FSR is the unpumped cavity free spectral range) is normalized by $\gamma$, the photon decay rate.

To vary the phase mismatch, the pump laser wavelength $\lambda_{\mathrm{P}}$ is tuned by selecting different longitudinal modes within the same transverse mode family, thereby tuning Brillouin shift $\Omega$. Since $\Omega$ is not directly measurable in the experiment, we instead obtained information on the phase mismatch using $\delta \omega$, which requires measurement of $\Delta \omega$, FSR and $\gamma$. The frequency difference $\Delta \omega$ is determined by measuring the beatnote of the pump and the SBL. At each pump wavelength, $\Delta \omega$ is slightly shifted by SBL power linearly and linear fitting has been applied to eliminate this effect. To determine the FSR at each pumping wavelength, the mode spectrum of the resonator is measured using a radio-frequency calibrated optical interferometer. The measured $\Delta \omega$ and FSR are summarized versus wavelength as the blue data points in Fig. 1b. The phase-matching condition occurs when FSR equals $\Delta \omega$ at around $1548 \mathrm{~nm}$ pump wavelength. $\gamma$ is determined by measurement of the cavity linewidth at each wavelength (equivalently, the total Q-factor of the resonator). The theoretical $\alpha$ factor as a function of wavelength is plotted in Fig. $1 \mathrm{~b}$ as red circles. The largest $\alpha$ factor is greater than 7 so that a fundamental linewidth enhancement of more than $1+7^{2}=50$ is expected at the largest detuning values.

A frequency discriminator method [5] is used to measure the noise spectrum of the two-sided white frequency noise spectral density $S_{\mathrm{w}}$ of the SBL. The fundamental noise component in $S_{\mathrm{w}}$, defined as $S_{\mathrm{F}}$, is related to the enhanced ST linewidth by $S_{\mathrm{F}}=\Delta \nu_{\mathrm{SBL}} /(2 \pi)$ and has inverse power dependence. Plots of $S_{\mathrm{W}}$ versus inverse power (Fig. 2a) reveal this power dependence, and the slope is equal to $S_{\mathrm{F}}$ normalized to an output power of $1 \mathrm{~mW}$. Linear fitting provides the slopes which are plotted versus wavelength in Fig. $2 b$. The corresponding minimum measured fundamental noise is about $S_{\mathrm{F}}=0.2 \mathrm{~Hz}^{2} / \mathrm{Hz}\left(\Delta v_{\mathrm{SBL}}=1.25 \mathrm{~Hz}\right)$ near the gain center. And the maximum fundamental noise is more than $S_{\mathrm{F}}=10 \mathrm{~Hz}^{2} / \mathrm{Hz}\left(\Delta v_{\mathrm{SBL}}=63 \mathrm{~Hz}\right)$, corresponding to $50 \times$ noise enhancement at the largest mismatch detunings. Theoretical calculations of $S_{\mathrm{F}}$ are provided as the green plot in Fig. $2 \mathrm{~b}$, where no free parameters are used. There is overall good agreement with the measured linewidth values. The conventional $S_{0}=\Delta v_{0} /(2 \pi)$ (with $\alpha=0$ ) is also plotted for comparison.

In summary, we have studied the linewidth enhancement factor in a Brillouin laser, where a modified linewidth formula incorporating $\alpha$ is tested experimentally. Phase mismatch induced by tuning the pumping wavelength leads to $50 \times$ linewidth enhancement. The results highlight the importance of proper phase matching in SBL operation and will be important in narrow-linewidth applications.

We acknowledge support from AFOSR (FA9550-18-1-0353) and the Caltech Kavli Nanoscience Institute.

\section{References}

1. J. Li, H. Lee, T. Chen and K. J. Vahala, Opt. Express 20, 20170-20180 (2012).

2. M. Lax, Phys. Rev. 157, 213-231 (1967).

3. C. Henry, IEEE J. Quantum Electron. 18, 259-264 (1982).

4. Y. R. Shen and N. Bloembergen, Phys. Rev. 137, A1787-A1805 (1965).

5. H. Ludvigsen, M. Tossavainen and M. Kaivola, Opt. Commun. 155, 180-186 (1998). 